\title{
Review of: "Why are they not accessing it? User barriers to clinical information access"
}

\author{
Fida Dankar ${ }^{1}$ \\ 1 United Arab Emirates University
}

Potential competing interests: The author(s) declared that no potential competing interests exist.

The paper reports on an initiative by the Medical Library Association (MLA) to identify the barriers that healthcare providers face when accessing information in the clinical environment. Library professional and "industry partners" are brought together, and divided into working groups to define and understand how clinicians discover and access information, the main barriers they face in their quest along with a list of potential solutions.

The report is well written, it describes and analyzes eight impediments (referred to as pain points) that prevent/complicates healthcare providers' access to critical information. It provides general guidelines on how to address each of the defined points, and initiates calls for action to relevant industry partners to reflect on the defined problems/solutions. More importantly, the report sheds some light on the role that (health) library professionals can play to help clinicians in defining and overcoming barriers to information access.

The report could benefit from multiple enhancements:

- The role and scope of work for each of the stakeholders (library professionals, users and industry partners) is not well defined (particularly, the role/scope of work of library professionals along with the challenges they face)

- The references used in the introduction to establish the need for information professionals are relatively old (1985, 1995 and 2014). The Amount of information available, as well the technology used to retrieve information are fast changing which necessitates more recent studies.

- The paper presents some high-level solutions (ideas) for some of the pain points. However, the challenges in implementing these solutions were not discussed.

- It is not clear form the paper whether one or two working groups were formed, nor the size of the groups, how they were selected, or their background.

- The role of the EUAB (vs. working groups) in determining and shaping the final pain points is not clear.

- The paper cites "lack of time" as one of the major pain points when trying to access information. This creates an additional constraint on any provided solution. A good discussion on how to design efficient solutions under this constraint could enrich the discussion. 
The general MLA initiative would benefit from the inclusion of artificial intelligence experts (along side librarians and clinicians) to discuss the role that artificial intelligence can possibly play in enhancing information search, determining what information can be trusted, as well as designing user-friendly mechanisms for information sharing. 